\title{
Neurogenesis in the Adult Brain
}

\author{
Fred H. Gage \\ Laboratory of Genetics, The Salk Institute, La Jolla, California 92037
}

A milestone is marked in our understanding of the brain with the recent acceptance, contrary to early dogma, that the adult nervous system can generate new neurons. One could wonder how this dogma originally came about, particularly because all organisms have some cells that continue to divide, adding to the size of the organism and repairing damage. All mammals have replicating cells in many organs and in some cases, notably the blood, skin, and gut, stem cells have been shown to exist throughout life, contributing to rapid cell replacement. Furthermore, insects, fish, and amphibia can replicate neural cells throughout life. An exception to this rule of self-repair and continued growth was thought to be the mammalian brain and spinal cord. In fact, because we knew that microglia, astrocytes, and oligodendrocytes all normally divide in the adult and respond to injury by dividing, it was only neurons that were considered to be refractory to replication. Now we know that this long accepted limitation is not completely true, because there are two rather discrete areas of the brain, the dentate gyrus of the hippocampal formation and the subventricular zone and its projection through the rostral migratory stream to the olfactory bulb, which can generate new neurons.

Although the discovery that there are limited areas of the brain where neurons do regenerate is important, it also deepens our curiosity as to why it is that most neurons in fact cannot replicate. One reason why neuronal replication in the adult was considered unlikely was the complexity of most neurons; with their highly branched dendrites and polysynaptic axonal combinations, they were considered to be terminally differentiated and unable to re-enter the cell cycle and divide, strictly from a mechanistic view. Another problem was conceptual. If neurons were able to divide, how would the newly created cells with their new dendrites, axons, and synapses, functionally integrate into the brain without disrupting existing circuits? With the dominant theories of brain function being based on a computer analogy with fixed circuits, it did not seem at all logical that adult brain circuitry could be continually adding new components.

Several recent discoveries and refinements of our conception of the brain have converged to help make the concept of neurogenesis in the adult brain more easily acceptable.

The mechanical problem of accommodating the dividing neurons has been overcome by the discovery that neural stem cells exist throughout life in the adult brain and can renew and give rise to new neurons, astrocytes, and oligodendrocytes, just as in the developing brain. This was first shown from the subventricular zone (Reynolds and Weiss, 1992; Richards et al., 1992) and then in the dentate gyrus of the hippocampus (Gage et al., 1995; Palmer et al., 1997), and in most structures of the brain examined

Correspondence should be addressed to Dr. Gage at the above address. E-mail: gage@salk.edu.

Copyright (C) 2002 Society for Neuroscience $\quad 0270-6474 / 02 / 220612-02 \$ 15.00 / 0$
(Palmer et al., 1995; Shihabuddin et al., 2000), even where no neurons existed (Palmer et al., 1999; Kondo and Raff, 2000).

These studies demonstrating that cells with stem cell properties exist in the adult brain were conducted in vitro, so it is not clear whether these cultured cells have the same potential in vivo as in vitro. Nevertheless, they showed that we no longer had to consider that a complex neuron was required to divide for adult neurogenesis to occur. Now we know that these neurons can be generated from primitive cells, similar to what happens in development.

A second change in thinking was more gradual and conceptual, and is still not completely resolved. Almost as soon as the first computers were made, the analogy with the brain was accepted. The model of the brain as a computer, with hard-wired connections that are adaptive and flexible mostly with respect to the software and with information that is distributed over the hardwired network still dominates.

The pervasive theory of adaptability or plasticity was provided by Hebb (1949), who postulated that plasticity, and adaptability could be accomplished by "strengthening synapses" without requiring structural reorganization.

A decade or so after the ascendance of the computer model of brain circuitry, Altman (1962) made his original claim of neurogenesis, and a theory of structural plasticity began to emerge. Soon afterward, examples of axonal elongation and synaptic reorganization in response to injury were provided (Raisman, 1969; Moore et al., 1971; Lynch et al., 1973). Within the same time period, investigators demonstrated that experience alone could promote structural changes in presynaptic and postsynaptic elements (Greenough et al., 1978).

The willingness to accept adult neurogenesis was further enhanced by the convincing evidence that fetal tissue could be grafted in the adult intact brain. Even more convincing was the evidence that the damaged adult brain and spinal cord allowed these newly grafted cells to survive and differentiate (Bjorklund and Gage, 1985). The grafted cells could also receive and send connections and release transmitter in a behavior- dependent manner in the adult damaged brain (Dunnett and Bjorklund, 1994)

The stage has been now set for accepting the ability of stem cells in the adult neuronal system to give rise to neurons. In addition, the damaged adult brain appears to retain enough potential to accept, if not participate in, the differentiation of developing cells into functional neurons.

The authors of the following mini-reviews are significant contributors to the current re-emergence of adult neurogenesis. There are several themes that the authors have independently identified. First, they each have presented a historical perspective that helps explain how their view of this field developed. Although it is likely that a field as young as this is searching for it 
origins, it is likely that it will take more time for the field to reveal its historically relevant origins.

A second theme that emerges from these reviews is the concern over the techniques that are used to document the neurogenic events that are being reviewed and examined. Each of these authors has contributed to the continued development of the tools, but it is clear that more and better methods are needed to quantitatively document the birth, proliferation, and migration of the new neurons and provide evidence for the extent to which new neurons functionally integrate into the adult brain. Will better techniques reveal more neurogenesis in more areas?

Another theme that all of the authors developed and acknowledge is the remarkable extent to which the environment in which the adult animal finds itself, and in particular how the organism behaves in that environment, can affect the birth rate and fate of the newly born cells. Is it possible that, with the appropriate behavioral and environmental stimulation and more accurate techniques, neurogenesis will be revealed or induced in other regions previously considered non-neurogenic? Or will they give insight into the mechanisms that limit neurogenesis in different brain regions, and by so doing, lead to ways to circumvent those mechanisms?

A series of questions related to the identity of the endogenous cells as they transition from the most primitive stem-like cell to the most mature neuron is currently being very intensely investigated and debated. The answers to those questions may surprise us and force a re-evaluation of our current understanding of stem cell or precursor biology. Clearly there are lessons to be learned from developmental biology, in which the process and mechanisms of neurogenesis have been the topic of intense investigation for decades. A fundamental question raised in the last review asks to what extent the mechanisms of neurogenesis in development and in the adult are the same. Given the wealth of knowledge available in developmental biology, this should be a tractable problem.

Finally, the two unresolved questions that all agree on are: (1) why does adult neurogenesis persist in only a few identified brain regions and, even more perplexing, (2) what is the function that underlies this residual structural plasticity?

Solutions to the first question will likely require a comparative approach, which considers the evolutionary development of these structures. The second question of function will likely require a reconceptualization of neural plasticity that incorporates structural plasticity into its theoretical framework. A more complete answer to either of the questions will require a better understanding of the normal function of the hippocampal formation and olfactory bulb and their local circuits. Perhaps a better understanding of the process of neurogenesis within these structures will elucidate more completely the functions these more global structures subserve.

The series begins with Pasko Rakic and Elizabeth Gould discussing the strengths and limitations of the methodologies used to identify cells undergoing neurogenesis in the mammalian dentate gyrus of the hippocampus. Fernando Nottebohm describes the perspective from work on avian brains, which have shown prominent abilities for neurogenesis in the adult. Arturo AlvarezBuylla discusses a system in the mammalian brain, the anterior migratory stream, that continues to generate new neurons during adult olfactory bulb, and he discusses recent work on the origin and phenotype of the initiating cell. Gerd Kempermann returns to neurogenesis in the dentate gyrus and focuses on the role of behavioral activity on the regulation of adult neurogenesis, and he discusses the functional significance of this process in the hippocampus. Finally, Chris Kintner summarizes the key elements and principles of neurogenesis that have been revealed from the extensive developmental neurobiology literature, and he makes parallels with adult neurogenesis. Importantly, this last review leads to some specific hypothesis and prediction when determining whether adult and developmental neurogenesis are equivalent.

\section{REFERENCES}

Altman J (1962) Are neurons formed in the brains of adult mammals? Science 135:1127-1128.

Bjorklund A, Gage FH (1985) Neural grafting in animal models of neurodegenerative diseases. In: Hope for a new neurology (Nottebohm F, ed), pp 53-81. New York: Ann NY Acad Sci.

Dunnett SB, Bjorklund A (1994) Functional neural transplantation. New York: Raven.

Gage FH, Coates PW, Palmer TD, Kuhn HG, Fisher LJ, Suhonen JO, Peterson DA, Suhr ST, Ray J (1995) Survival and differentiation of adult neuronal progenitor cells transplanted to the adult brain. Proc Natl Acad Sci USA 92:11879-11883.

Greenough WT, West RW, DeVoogd TJ (1978) Postsynaptic plate perforations: changes with age and experience in the rat. Science 202:1096-1098

Hebb DO (1949) The organization of behavior. New York: Wiley.

Kondo T, Raff M (2000) Oligodendrocyte precursor cells reprogrammed to become multipotential CNS stem cells. Science 289:1754-1757.

Lynch G, Deadwyler D, Cotman CW (1973) Post lesion axonal growth produces permanent functional connections. Science 180:1364-1366.

Moore RY, Bjorklund A, Stenevi U (1971) Plastic changes in the adrenergic innervation of the rat septal area in response to denervation. Brain Res 33:13-35.

Palmer TD, Ray J, Gage FH (1995) FGF-2-responsive neuronal progenitors reside in proliferative and quiescent regions of the adult rodent brain. Mol Cell Neurosci 6:474-486.

Palmer TD, Markakis EA, Willhoite AR, Safar F, Gage FH (1999) Fibroblast growth factor 2 activates a latent neurogenic program in neural stem cells from diverse regions of the adult CNS. J Neurosci 19:8487-8497.

Palmer TD, Takahashi J, Gage FH (1997) The adult rat hippocampus contains primordial neural stem cells. Mol Cell Neurosci 8:389-404.

Raisman G (1969) Neuronal plasticity in the septal nuclei of the adult rat. Brain Res 14:25-48.

Reynolds BA, Weiss S (1992) Generation of neurons and astrocytes from isolated cells of the adult mammalian central nervous system. Science 255:1707.

Richards LJ, Kilpatrick TJ, Bartlett PF (1992) De novo generation of neuronal cells from the adult mouse brain. Proc Natl Acad Sci USA 89:8591-8595.

Shihabuddin LS, Horner PJ, Ray J, Gage FH (2000) Adult spinal cord stem cells generate neurons after transplantation in the adult dentate gyrus. J Neurosci 20:8727-8735. 